\title{
Using biomarkers to detect oral cancer holds potential for saving lives when the cancer is
}

\section{most curable}

\author{
"A variety of genetic and molecular signatures of precancerous lesions and OSCC have \\ been identified as tumor biomarkers. However, these biomarkers have, so far, not \\ gained any use in routine diagnosis and risk assessment of oral cancer..."
}

\section{KEYWORDS: biomarker = carcinoma in situ " defensins " leukoplakia = oral cancer}

Oral squamous cell carcinoma (OSCC) comprises approximately $3-5 \%$ of all malignancies in the USA, corresponding to 40,490 new cases and approximately 10,000 deaths each year [1-3]. Despite significant advances in therapeutics and early diagnosis, the expected 5 -year relative survival rate of oral cancer has improved only marginally over the past decade [1-3]. Early diagnosis of oral cancer plays a key role in disease progression, treatment response, and ultimately, quality of life and patient survival. Therefore, biomarkers for risk prediction of oral cancer hold much promise in this respect. Various oral lesions, including leukoplakia, erythroplakia, lechen planus and submucous fibrosis, are considered potentially malignant oral disorders that may contain precancerous cells, which could malignantly transform [4]. Currently, these lesions are treated surgically, with or without cellular and tissue changes (dysplasia). However, it is unknown if surgery can really prevent transformation into OSCC. Although the term "potentially malignant disorders" was recommended by the WHO to describe precancerous lesions, the clinical and histological features alone cannot accurately predict whether these precancers of the oral mucosa remain stable, regress or progress to malignancy [5]. Furthermore, evaluation of an asymptomatic patient for early-stage cancer, based on its physical features alone, is frequently compromised because malignant and benign lesions may not be clinically distinguishable [6]. Consequently, approximately $60 \%$ of oral cancers are advanced by the time they are detected, and approximately $15 \%$ of patients have another cancer in a nearby area such as the larynx, esophagus or lungs $[7,8]$. Therefore, there is a need to identify and use molecular biomarkers to evaluate individuals with potentially malignant disorders who are at a high risk of developing OSCC and those with early-stage malignant lesions.
A variety of genetic and molecular signatures of precancerous lesions and OSCC have been identified as tumor biomarkers. However, these biomarkers have, so far, not gained any use in routine diagnosis and risk assessment of oral cancer [5,9]. Malignant transformation or progression to OSCC requires multiple genetic alternations and involves a variety of stromal cells, such as macrophages, in the tumor microenvironment [10]. Therefore, identification of molecular markers that can predict disease progression and techniques that can readily use such biomarkers in routine practices will improve the management of these disorders.

\section{Potentially malignant disorders \& oral cancer}

Potentially malignant disorders are currently referred to as all clinical entities that carry a risk of cancer with widespread anatomical distribution. In this regard, leukoplakia is a clinical presentation that is defined by the WHO as a "white patch or plaque that cannot be characterized clinically or pathologically as any other disease" and is, by far, the most common precancer, accounting for over $80 \%$ of potentially malignant oral disorders $[4,11,12]$. In addition, leukoplakia is also a relatively common oral lesion, ranging from 1 to $5 \%$ in the general population [12]. However, it does not have the highest malignant transformation risk among precancerous lesions. Oral erythroplakia, a relatively rare (reported incidence: $0.02-0.80 \%$ ) red lesion of the oral cavity that cannot be removed, has a much higher malignant transformation risk than leukoplakia; up to $50 \%$ of these lesions are invasive OSCC and $40 \%$ are carcinoma in situ $[12,13]$. Risk assessment of precancerous lesions, such as leukoplakia, is currently performed based on the histological criteria of cellular proliferation,

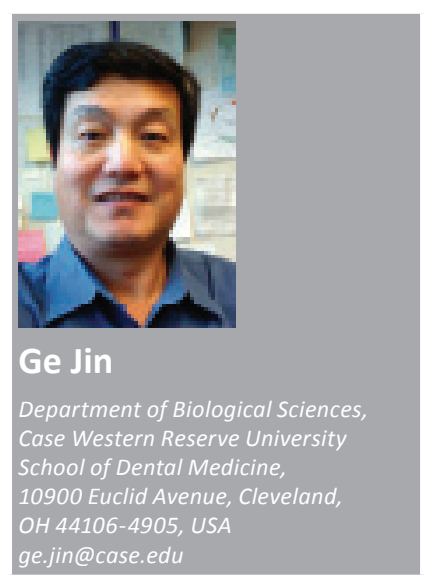

future medicine $^{\text {pint }}$ fs 
maturation of the epithelium and cellular atypia, and graded as mild, moderate and severe dysplasia. However, lack of objectivity of the established histological criteria and difficulties in predicting the outcome of individual cases calls for the identification and practical application of reliable biomarkers [5].

\section{Molecular biomarkers \& histological assessment}

Alternations in genes and pathways that regulate cellular signaling, cell cycle, differentiation, apoptosis, genomic stability, motility, angiogenesis and metastasis are significantly associated with development and progression of a potentially malignant disorder to OSCC $[5,9]$. Aberrant expression and function of molecules involved in these signaling networks have been considered as biomarkers for risk assessment of malignant transformation. These biomarkers, including oncogenes (EGF receptor $[E G F R]$, $E G F, R A S$ and $M Y C$ ), tumor suppressor genes $(p 53,-21,-27$ and -16$)$ and proliferation markers (Ki67 and proliferating cell nuclear antigen), have been analyzed from fixed biopsies, on homogenized tissue samples and in body fluid, such as saliva. However, spatial and temporal presentation of these molecules in precancerous lesions and OSCC often matches up with histological assessment, which may limit their use as biomarkers in routine practice. For example, enhanced expression and activation of the EGFR family and its ligands, EGF and TGF- $\alpha$, have been associated with precancerous leukoplakia [5,11]. EGFR is detected in proliferative cells of the basal layer in normal oral mucosa and leukoplakia with mild, moderate and severe dysplastic lesions $[5,11,13]$. Interestingly, the pattern of EGFR expression in dysplastic leukoplakia coincides with the histological assessment of dysplasia in these lesions.

\section{"Although the term "potentially malignant disorders" was recommended by the WHO to describe precancerous lesions, the clinical and histological features alone cannot accurately predict whether these precancers of the oral mucosa remain stable, regress or progress to malignancy."}

Dysfunction of the $p 53$ pathway, frequently through mutations and loss of heterozygosity, has been associated with early head and neck carcinogenesis, and can be detected in precancerous lesions [5]. Mutant $\mathrm{p} 53$ proteins have a prolonged half-life. Therefore, they are readily detected in biopsies using immunohistochemistry [5]. Similar to the pattern of EGFR expression in dysplasia, the expression of $\mathrm{p} 53$ protein can be identified in dysplastic lesions with features that recapitulate histological evaluation $[5,14,15]$. Therefore, these biomarkers may serve as adjuncts to histological assessment of malignant transformation. However, their application in routine practice are limited and not defined.

\section{Human $\beta$-defensins as biomarkers for OSCC}

Recent progress in molecular oncology and cancer biology has demonstrated that accumulation of genetic alterations drives the progression of dysplastic/neoplastic lesions to highly malignant derivatives, a process not only involving tumor cells, but also many other nonmalignant cell types, including innate and adaptive immune cells, fibroblasts, epithelial cells and endothelial cells, in the tumor microenvironment [16,17]. Tumor cell-derived molecules, such as chemokines and proangiogenic factors, play a critical role in initiation, maintenance and expansion of the protumor environment. Therefore, these molecules may have the potential to serve as biomarkers for routine application.

\section{“...accumulation of genetic alterations drives the progression of dysplastic/neoplastic lesions to highly malignant derivatives, a process not only involving tumor cells, but also many other nonmalignant cell types, including innate and adaptive immune cells, fibroblasts, epithelial cells and endothelial cells, in the tumor microenvironment..."}

Kawsar et al. have demonstrated that human $\beta$-defensins (hBDs) are spatiotemporally expressed in normal, dysplasia, carcinoma in situ and OSCC biopsies [18]. hBDs are small secreted peptides, originally identified from the plasma of patients with renal disease and from psoriatic skin lesions as antimicrobial molecules of innate immunity [19-21]. Oral epithelia express hBD-1, -2 and -3 . hBD-1 appears to be constitutively expressed in oral epithelial cells [22]. In oral epithelial and gastric cells, hBD-2 induction has been demonstrated to require activation of NF- $\kappa$ B transcription factor [23,24], while hBD-3 expression is induced by EGF via MEK1/2, protein kinase C, PI3K and p38 MAPK signaling cascades in oral epithelial cells [18]. In normal oral epithelia, co-expression of hBD-1 and -2 is primarily associated with differentiated epithelial cells in the superficial layers, whereas hBD-3 
is predominantly produced by proliferative cells in the basal layer $[18,25]$. In moderate dysplasia, tissue distribution of hBD-2 and -3 is correlated with dysplastic cells in the lesion [18]. However, in carcinoma in situ biopsies, the entire lesion site expresses hBD-3, with little to no expression of hBD-1 and $-2[10,18]$. Overexpression of hBD-3 in oral cancer has also been confirmed by a clinical study [26]. Furthermore, hBD-3 promotes macrophage infiltration and tumorigenicity in nude mice, recruits monocytes via the chemokine receptor CCR 2 and stimulates the expression of tumor-promoting cytokines in macrophages $[10,18]$. These results suggest that the overexpression of hBD-3 and loss of hBD-2 in neoplastic cells of oral carcinoma in situ may serve as a signature of oncogene activation, leading to cellular transformation and precancerous lesions. In addition, hBD-3 is a secreted peptide and, once produced by precancerous lesions, it may enter the circulation and be readily detected in blood, saliva and other body fluids. Therefore, a noninvasive, moderate-cost screening protocol can be developed for risk assessment of malignant transformation.

\section{"Recent progress in high-throughput techniques, including the profiling of gene expression (microarray), detection of single-nucleotide changes of preselected genes, miRNA analysis and salivary proteomics, will greatly promote identification and development of novel biomarkers..."}

Oral squamous cell carcinoma is initiated from stem cells of the basal layer of the epithelium that have acquired genetic alterations, such as activating mutations or amplification of oncogenes, as well as inactivation of tumor suppressor genes [27-29]. Although aberrant expression of these genes are etiologically relevant to oral carcinogenesis, dysfunctional regulation of many other molecules, which are either modulated by or interact with those genes, play crucial roles in promoting malignant transformation and cancer progression. Therefore, identification of biomarkers that are involved in different aspects of oral carcinogenesis will significantly increase sensitivity, predictability and reproducibility in risk assessment of malignant transformation.

Recent progress in high-throughput techniques, including the profiling of gene expression (microarray), detection of single-nucleotide changes of preselected genes, miRNA analysis and salivary proteomics, will greatly promote identification and development of novel biomarkers that distinguish normal oral epithelia from potentially malignant lesions and earlystage oral cancer. Further validation of these biomarkers and application in combination with routine histological studies will lead to improved diagnostic approaches and therapeutic strategies when the cancer is most curable.

\section{Financial \& competing interests disclosure \\ The author has no relevant affliations or financial involve- ment with any organization or entity with a financial inter- est in or financial conflict with the subject matter or materi- als discussed in the manuscript. This includes employment, consultancies, honoraria, stock ownership or options, expert testimony, grants or patents received or pending, or royalties.}

No writing assistance was utilized in the production of this manuscript.

\section{Bibliography}

1 Jemal A, Siegel R, Ward E et al.: Cancer statistics, 2006. CA Cancer J. Clin. 56, 106-130 (2006).

2 Khuri FR, Lee JJ, Lippman SM et al.: Randomized Phase III trial of low-dose isotretinoin for prevention of second primary tumors in stage I and II head and neck cancer patients. J. Natl Cancer Inst. 98, 441-450 (2006).

3 Gasparotto D, Maestro R: Molecular approaches to the staging of head and neck carcinomas (review). Int. J. Oncol. 31, 175-180 (2007).

4 Mithani SK, Mydlarz WK, Grumbine FL et al:: Molecular genetics of premalignant oral lesions. Oral Dis. 13, 126-133 (2007).
5 Pitiyage G, Tilakaratne WM,

Tavassoli M et al.: Molecular markers in oral epithelial dysplasia: review. J. Oral Pathol. Med. 38, 737-752 (2009).

6 Rethman MP, Carpenter W, Cohen EE et al.: Evidence-based clinical recommendations regarding screening for oral squamous cell carcinomas. J. Am. Dent. Assoc. 141, 509-520 (2010).

7 Gonsalves WC, Chi AC, Neville BW: Common oral lesions: Part II. Masses and neoplasia. Am. Fam. Physician 75, 509-512 (2007).

8 Weinberg MA, Estefan DJ: Assessing oral malignancies. Am. Fam. Physician 65, 1379-1384 (2002).
9 Molinolo AA, Amornphimoltham P, Squarize $\mathrm{CH}$ et al.: Dysregulated molecular networks in head and neck carcinogenesis. Oral Oncol. 45, 324-334 (2009).

10 Jin G, Kawsar HI, Hirsch SA et al.: An antimicrobial peptide regulates tumorassociated macrophage trafficking via the chemokine receptor CCR2, a model for tumorigenesis. PLoS ONE 5, e10993 (2010).

11 Taoudi Benchekroun M, Saintigny P, Thomas SM et al.: Epidermal growth factor receptor expression and gene copy number in the risk of oral cancer. Cancer Prev. Res. (Phila.) 3, 800-809 (2010).

12 Kademani D: Oral cancer. Mayo Clin. Proc. 82, 878-887 (2007). 
13 Reichart PA, Philipsen HP: Oral erythroplakia - a review. Oral Oncol. 41, 551-561 (2005).

14 Reibel J: Prognosis of oral pre-malignant lesions: significance of clinical, histopathological, and molecular biological characteristics. Crit. Rev. Oral Biol. Med. 14, 47-62 (2003).

15 Prado SM, Cedrun JL, Rey RL et al.: Evaluation of COX-2, EGFR, and $\mathrm{p} 53$ as biomarkers of non-dysplastic oral leukoplakias. Exp. Mol. Pathol. 89, 197-203 (2010).

16 Allavena P, Sica A, Solinas G et al:: The inflammatory micro-environment in tumor progression: the role of tumorassociated macrophages. Crit. Rev. Oncol. Hematol. 66, 1-9 (2008).

17 Grivennikov SI, Greten FR, Karin M: Immunity, inflammation, and cancer. Cell 140, 883-899 (2010).

18 Kawsar HI, Weinberg A, Hirsch SA et al.: Overexpression of human $\beta$-defensin-3 in oral dysplasia: potential role in macrophage trafficking. Oral Oncol. 45(8), 696-702 (2009).
19 Harder J, Bartels J, Christophers E et al.: A peptide antibiotic from human skin. Nature 387, 861 (1997).

20 Harder J, Bartels J, Christophers E et al.: Isolation and characterization of human $\beta$-defensin-3, a novel human inducible peptide antibiotic. J. Biol. Chem. 276, 5707-5713 (2001).

21 Bensch KW, Raida M, Magert HJ et al.: hBD-1: a novel $\beta$-defensin from human plasma. FEBS Lett. 368, 331-335 (1995).

22 Jurevic RJ, Bai M, Chadwick RB et al.: Single-nucleotide polymorphisms (SNPs) in human $\beta$-defensin 1: high-throughput SNP assays and association with Candida carriage in Type I diabetics and nondiabetic controls. J. Clin. Microbiol. 41, 90-96 (2003).

23 Chung WO, Dale BA: Differential utilization of nuclear factor- $\mathrm{KB}$ signaling pathways for gingival epithelial cell responses to oral commensal and pathogenic bacteria. Oral Microbiol. Immunol. 23, 119-126 (2008).

24 Boughan PK, Argent RH, Body-Malapel M et al: : Nucleotide-binding oligomerization domain-1 and epidermal growth factor receptor: critical regulators of $\beta$-defensins during Helicobacter pylori infection.

J. Biol. Chem. 281, 11637-11648

(2006).

25 Lu Q, Samaranayake LP, Darveau RP et al.: Expression of human $\beta$-defensin- 3 in gingival epithelia. J. Periodontal Res. 40, 474-481 (2005).

26 Kesting MR, Loeffelbein DJ, Hasler RJ et al:: Expression profile of human $\beta$-defensin 3 in oral squamous cell carcinoma. Cancer Invest. 27, 575-581 (2009).

27 Choong NW, Cohen EE: Epidermal growth factor receptor directed therapy in head and neck cancer. Crit. Rev. Oncol. Hematol. 57, 25-43 (2006).

28 Choi S, Myers JN: Molecular pathogenesis of oral squamous cell carcinoma: implications for therapy. J. Dent. Res. 87, 14-32 (2008).

29 Mayo MW, Wang CY, Cogswell PC et al: Requirement of NF- $\kappa \mathrm{B}$ activation to suppress $\mathrm{p} 53$-independent apoptosis induced by oncogenic Ras. Science 278, 1812-1815 (1997). 